\title{
Erratum
}

\section{The Politics of Anti-Social Behaviour}

\section{Peter Squires}

British Politics (2008) 3, 580. doi:10.1057/bp.2008.28

Correction to: British Politics (2008) 3, 300, doi:10.1057/bp.2008.16

We would like to rectify an editorial mistake that appeared in our previous issue. In Peter Squires' paper 'The Politics of Anti-Social Behaviour', it was wrongly stated that the Chair of the Youth Justice Board was 'Rhodri Morgan' when it should have been 'Rod Morgan'. We would like to apologise for this error and the Editors of British Politics are happy to make this correction. 